\title{
GMR
}

\section{Synthesis and structure-activity relationship of oleanolic mono- or di-glycosides against Magnaporthe oryzae}

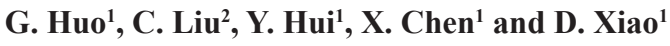 \\ ${ }^{1}$ Institute of Bioresource Conservation and Utilization/College of Bioscience \\ and Engineering, Jiangxi Agricultural University, Nanchang, China \\ ${ }^{2}$ College of Pharmacy, Gannan Medical University, Ganzhou, China \\ Corresponding author: G. Huo \\ E-mail: hgh3828079@sohu.com
}

Genet. Mol. Res. 15 (3): gmr.15038998

Received July 21, 2016

Accepted August 1, 2016

Published September 23, 2016

DOI http://dx.doi.org/10.4238/gmr.15038998

Copyright $(C 2016$ The Authors. This is an open-access article distributed under the terms of the Creative Commons Attribution ShareAlike (CC BY-SA) 4.0 License.

\begin{abstract}
Saponins are naturally-occurring units with broad diversity and are usually recognized as phytoanticipins. In order to develop new saponin chemical entities with high activity against Magnaporthe oryzae, we selected oleanolic acid (OA), which has wide natural distribution and rich content in plants. We used the ability of OA to act as an aglycone for glycosylation to obtain information on the structure-activity relationship (SAR) for rational molecular pesticide design. Oleanolic mono- or di-glycosides were synthesized at either the $\mathrm{C}_{3}$-hydroxy and/or $\mathrm{C}_{28}$-carboxyl position, using trichloroacetimidate or glycosyl bromide donors, respectively. Structures were confirmed by $\left[{ }^{1} \mathrm{H}\right]-,\left[{ }^{13} \mathrm{C}\right]-N M R$. Furthermore, the activity of the synthesized glycosides against $M$. oryzae was assessed in vitro, based on the mycelium growth rate. The twenty five oleanolic mono- or di-glycosides
\end{abstract}

Genetics and Molecular Research 15 (3): gmr.15038998 
comprised fourteen saponins with 3-monosaccharide residue 1a-1n, six saponins with 28 -monosaccharide residue $2 \mathrm{a}-2 \mathrm{f}$, and five saponins with 3, 28-monosaccharide residue 3a-3e; all showed different activities against $M$. oryzae according to their different structures. We concluded that the optimal oleanolic mono- and di-glycoside structure for activity against $M$. oryzae is a $\mathrm{C}_{3}$ connection of a hexose such as mannose, galactose, or glucose, in combination with a $\mathrm{C}_{28}$ connection to a small group such as allyl or a $\mathrm{C}_{3}$ connection to a pentose accompanied by a larger group such as another pentose or heptenyl at $\mathrm{C}_{28}$.

Key words: Oleanolic mono-glycosides; Oleanolic di-glycosides; Saponins; Magnaporthe oryzae; Structure-activity relationship

\section{INTRODUCTION}

Rice blast is caused by the fungus Magnaporthe oryzae and is the most prevalent disease in rice (Oryza sativa). The disease results in an average loss of about 157 million tons of rice per year worldwide, an amount that would be enough to feed 60 million people in one year (Shi and Wang, 2008). To control rice blast, major efforts have been made to develop disease-resistant varieties of rice. To date, over 85 blast-resistance genes have been identified, 20 of which have been cloned (Sun et al., 2013). However, owing to the pathogen's high adaptive variability, these genes do not provide permanent resistance to rice blast and typically last only 2-3 seasons (Normile, 2009). Furthermore, the resistant varieties often result in a lower quality of rice (Fukuoka et al., 2009). Rice species and cultivar diversity in ecological management are also being explored (Zhu et al., 2000; Han et al., 2013), but chemical control is still an indispensable means for controlling rice blast. Unfortunately, the chemicals used to control rice blast in the field, such as tricyclazole, have resulted in environmental residues (Zhou et al., 2013) and in some cases, isoprothiolaneresistant isolates of M. oryzae have emerged (Zhang et al., 2013). Thus, there is high demand for novel natural products with unique structures, modes of action, and high environmental safety, which can counteract the high adaptive variability of rice blast.

Saponins are natural phytoanticipins. Preliminary research has shown that schimasuoside, a new oleanane-type saponin from Schima superba, has a strong inhibitory activity against $M$. oryzae (Huo et al., 2014) and also has the potential to control rice blast in the field (Tan et al., 2014). Its aglycone structure 21,22-di- $O$-angeloyl- $\mathrm{R}_{1}$-barrigenol-3 $\beta, 15 \alpha, 16 \beta$-trihydroxyl17-hydroxymethyl-21,22-di- $O$-angeloyl-olea-12-en) is similar to that of oleanolic acid (OA; $3 \beta$-hydroxy-olea-12-en-28-oic acid). OA has two glycosylation sites, $\mathrm{C}(3)-\mathrm{OH}$ and $\mathrm{C}(28) \mathrm{OOH}$, which can be glycosylated to form 3-monoglycosides, 28-monoglycosides, and 3,28-diglycosides. Glycosylated saccharides may originate from commercially available D-glucose, D-galactose, D-xylose, D-arabinose, D-mannose, L-rhamnose, and maltose. OA is a favorable raw material that can be obtained from a wide number of sources at low cost. Moreover, glycosylation of OA can improve its weak aqueous solubility and bioavailability (Ghanbari et al., 2012). Therefore, it is of considerable interest to utilize OA as an initial aglycone to synthesize saponins with inhibitory activity against rice blast.

Genetics and Molecular Research 15 (3): gmr.15038998 


\section{MATERIAL AND METHODS}

\section{General experimental procedures}

Thin-layer chromatography (TLC) was performed using silica gel $\mathrm{GF}_{254}$ plates and column chromatography was performed using silica gel (100-200 mesh). NMR spectra were obtained using a Bruker $400 \mathrm{MHz}$ NMR spectrometer. Chemical shifts $(\delta)$ were expressed as ppm and coupling constants (J) were expressed in Hz. Tetramethylsilane (TMS) was used as internal reference.

\section{Procedures for preparing the synthetic compounds}

\section{Preparation of bromoglycosides and trichloroacetimidates}

Monosaccharides (D-glucose, D-galactose, D-xylose, D-arabinose, D-mannose, L-rhamnose) were successively reacted by benzoylation, bromination to yield benzoylbromoglycoside-6 (Glc-6, Gal-6, Xyl-6, Ara-6, Man-6, Rha-6). Benzoyltrichloroacetimidate-8 (Glc-8, Gal-8, Xyl-8, Ara-8, Man-8, Rha-8) was obtained from benzoylbromoglycoside- 6 by hydrolysis and a trichloroacetic amine esterification reaction, according to previous reports (Zheng et al., 2005; Li et al., 2011).

\section{Synthesis of oleanolic 28-allyl ester (10a)}

Oleanolic acid ( $2 \mathrm{~g}, 4.38 \mathrm{mmol})$ was dissolved in dry $10 \mathrm{~mL}$ dimethyl formamide (DMF) and stirred, and then allyl bromide $(0.5 \mathrm{~mL}, 5.78 \mathrm{mmol})$ and 1,8-diazabicycloundec-7ene (DBU; $0.5 \mathrm{~mL}, 3.35 \mathrm{mmol}$ ) were added. Reaction completion was determined by TLC (4:1 petroleum ether-EtOAc) after stirring for $24 \mathrm{~h}$ at room temperature. The mixture was extracted using EtOAc and water. The EtOAc phase was dried using anhydrous sodium sulfate. The filtrate was concentrated and purified by silica gel column chromatography (4:1 petroleum ether-EtOAc) to afford oleanolic 28 -allyl ester (10a) $\left(1.74 \mathrm{~g}, 82.9 \%\right.$ yield) as a white amorphous solid. $\mathrm{R}_{\mathrm{f}}=$ 0.50 (4:1 petroleum ether-EtOAc in TLC). [ $\left.{ }^{1} \mathrm{H}\right] \mathrm{NMR}\left(400 \mathrm{MHz}, \mathrm{CDCl}_{3}\right) \delta 5.90(\mathrm{ddd}, \mathrm{J}=16.8$, $\left.10.7,5.5 \mathrm{~Hz}, 1 \mathrm{H},-\mathrm{CH}_{2}-\mathrm{CH}=\mathrm{CH}_{2}\right), \delta 5.29$ (brs, $\left.1 \mathrm{H}, \mathrm{H}-12\right), \delta 5.36-5.16\left(\mathrm{~m}, 2 \mathrm{H},-\mathrm{CH}_{2}-\mathrm{CH}^{-} \mathrm{CH}_{2}\right), \delta$ $4.53\left(\mathrm{~m}, 2 \mathrm{H},-\mathrm{CH}_{2}-\mathrm{CH}=\mathrm{CH}_{2}\right), \delta 3.21(\mathrm{~m}, 1 \mathrm{H}, \mathrm{H}-3), \delta 2.88(\mathrm{~d}, \mathrm{~J}=10.2 \mathrm{~Hz}, 1 \mathrm{H}, \mathrm{H}-18), \delta 1.98(\mathrm{~m}$, 1H,H-16-1), $\delta 1.87$ (m, 2H,H-11), $\delta 1.14$ (s, 3H,H-27), $\delta 0.99$ (s, 3H,H-25), $\delta 0.93$ (s, 3H,H-23), $\delta 0.90$ (s, 6H,H-29,H-30), $\delta 0.78$ (s, 3H,H-24), $\delta 0.73$ (s, 3H,H-26).

\section{Synthesis of oleanolic-28-allylation ester-3-glycosides (1a-1f) and oleanolic-3- glycosides (1i-1n)}

Oleanolic-28-allylation ester -3-O- $\beta$-D-glucopyranoside (1a): Glc-8 (0.72 g, 0.97 $\mathrm{mmol})$ and $10 \mathrm{a}(0.37 \mathrm{~g}, 0.74 \mathrm{mmol})$ and $0.5 \mathrm{~g}$ powdered 4 - $\AA$ molecular sieves $(0.50 \mathrm{~g})$ were stirred at room temperature in dry $5 \mathrm{~mL} \mathrm{CH}_{2} \mathrm{Cl}_{2}$. When the reaction temperature dropped to $0^{\circ} \mathrm{C}$, a solution of TMSOTf $(10 \mu \mathrm{L}, 0.055 \mathrm{mmol})$ was added dropwise and stirred for $2 \mathrm{~h}$ at room temperature. The reaction was quenched with triethylamine, filtered through Celite, and concentrated under reduced pressure. The crude residue was purified by silica gel column chromatography to yield oleanolic 3-O- $\beta$-D-2,3,4,6-benzoyl-glucopyranosyl-28-allyl ester (Glc-11) $\left(0.65 \mathrm{~g}, 80.6 \%\right.$ yield) as a white solid. $\mathrm{R}_{\mathrm{f}}=0.52$ (4:1 petroleum ether-EtOAc). Glc-11

Genetics and Molecular Research 15 (3): gmr.15038998 


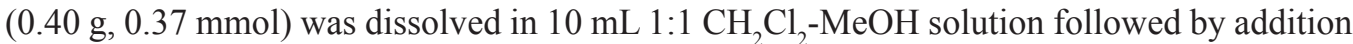
of $20 \mathrm{mg}$ sodium methoxide. The mixture was stirred for $2 \mathrm{~h}$ at room temperature. Completion of the reaction was determined by TLC $\left(10: 1, \mathrm{CH}_{2} \mathrm{Cl}_{2}-\mathrm{MeOH}\right)$, then the reaction mixture was filtered and concentrated under reduced pressure. The residue was purified by silica gel column chromatography $\left(20: 1 \rightarrow 10: 1, \mathrm{CH}_{2} \mathrm{Cl}_{2}-\mathrm{MeOH}\right)$ to give oleanolic-28-allyl-3-O- $\beta$-Dglucopyranoside (1a) $\left(0.21 \mathrm{~g}, 83.8 \%\right.$ yield) as a white solid. $\mathrm{R}_{\mathrm{f}}=0.26\left(10: 1 \mathrm{CH}_{2} \mathrm{Cl}_{2}-\mathrm{MeOH}\right)$.

Oleanolic-3-O- $\beta$-D-glucopyranoside (1i): Glc-8 (200 mg, $0.30 \mathrm{mmol})$ was dissolved in $4 \mathrm{~mL}$ anhydrous $\mathrm{MeOH}$, followed by addition of $10 \mathrm{mg}$ palladium dichloride. The reaction mixture was stirred for $24 \mathrm{~h}$ at room temperature then leached through diatomite. The filtrate was purified by silica gel column chromatography $\left(20: 1 \rightarrow 10: 1, \mathrm{CH}_{2} \mathrm{Cl}_{2}-\mathrm{MeOH}\right)$ to yield oleanolic-3-O- $\beta$-D -glucopyranoside (1i) ( $88 \mathrm{mg}, 0.14 \mathrm{mmol}, 46.7 \%$ yield) as a white solid. $\mathrm{R}_{\mathrm{f}}=0.21\left(10: 1 \mathrm{CH}_{2} \mathrm{Cl}_{2}-\mathrm{MeOH}\right) .\left[{ }^{1} \mathrm{H}\right] \mathrm{NMR}$ and $\left[{ }^{13} \mathrm{C}\right] \mathrm{NMR}$ data consistent with the assigned structure were the same as that in previous reports (Sha et al. 2008).

Compounds $1 \mathrm{~b}-1 \mathrm{f}$ were prepared according to the procedure described for $1 \mathrm{a}$, and compounds $1 \mathrm{j}-1 \mathrm{n}$ according to that described for $1 \mathrm{i}$. $\left[{ }^{1} \mathrm{H}\right]-$ and $\left[{ }^{13} \mathrm{C}\right]-\mathrm{NMR}$ data from $1 \mathrm{c}, 1 \mathrm{k}$, $1 \mathrm{~b}, 1 \mathrm{j}, 1 \mathrm{~d}, 1 \mathrm{l}, 1 \mathrm{n}$ are consistent with those in previous reports (Zang, 2004; Sha et al., 2008; Zhao et al., 2011). Oleanolic - 28 -allyl ester-3-O- $\beta$-D-galactopyranoside ( $1 \mathrm{c}): 58.4 \%$ yield, $\mathrm{R}_{\mathrm{f}}=0.26$ $\left(10: 1 \mathrm{CH}_{2} \mathrm{Cl}_{2}-\mathrm{MeOH}\right)$. Oleanolic 3-O- $\beta$-D-galactopyranoside (1k): $32.2 \%$ yield, $\mathrm{R}_{\mathrm{f}}=0.22\left(10: 1 \mathrm{CH}_{2} \mathrm{Cl}_{2}-\mathrm{MeOH}\right)$. Oleanolic -28 -allyl ester-3-O- $\beta$-D-xylopyranoside (1b): $76.4 \%$ yield, $\mathrm{R}_{\mathrm{f}}=0.36(10: 1$ $\left.\mathrm{CH}_{2} \mathrm{Cl}_{2}-\mathrm{MeOH}\right)$. Oleanolic 3-O- $\beta$-D-xylopyranoside $(1 \mathrm{j}): 45.0 \%$ yield, $\mathrm{R}_{\mathrm{f}}=0.33\left(10: 1 \mathrm{CH}_{2} \mathrm{Cl}_{2}-\mathrm{MeOH}\right)$. Oleanolic - 28 -allyl ester-3-O- $\alpha$-D-arabinopyranoside $(1 \mathrm{~d}): 83.5 \%$ yield, $\mathrm{R}_{\mathrm{f}}=0.38$ $\left(10: 1 \mathrm{CH}_{2} \mathrm{Cl}_{2}-\mathrm{MeOH}\right)$.

Oleanolic 3-O- $\alpha$-D-arabinopyranoside (11): $48.0 \%$ yield, $\mathrm{R}_{\mathrm{f}}=0.34\left(10: 1 \mathrm{CH}_{2} \mathrm{Cl}_{2}-\mathrm{MeOH}\right)$. Oleanolic - 28 -allyl ester- 3-O- $\beta$-D-mannopyranoside (1e): $33.8 \%$ yield, $\mathrm{R}_{\mathrm{f}}=0.25$ $\left.\left(10: 1 \mathrm{CH}_{2} \mathrm{Cl}_{2}-\mathrm{MeOH}\right) .{ }^{1} \mathrm{H}\right] \mathrm{NMR}\left(400 \mathrm{MHz}, \mathrm{CDCl}_{3}\right) \delta 5.90(\mathrm{ddd}, \mathrm{J}=22.6,10.8,5.6 \mathrm{~Hz}, 1 \mathrm{H})$, $5.33(\mathrm{dd}, 1 \mathrm{H}), 5.29(\mathrm{~s}, 1 \mathrm{H}), 5.20(\mathrm{dd}, \mathrm{J}=10.4 \mathrm{~Hz}, 1 \mathrm{H}), 4.98(\mathrm{~s}, 1 \mathrm{H}), 4.53(\mathrm{~m}, 2 \mathrm{H}), 3.95(\mathrm{~m}$, 1H), $3.85(\mathrm{~m}, 2 \mathrm{H}), 3.76(\mathrm{~d}, 1 \mathrm{H}), 3.65(\mathrm{~m}, 1 \mathrm{H}), 3.16(\mathrm{~d}, \mathrm{~J}=9.0 \mathrm{~Hz}, 1 \mathrm{H}), 2.88(\mathrm{~d}, \mathrm{~J}=12.7 \mathrm{~Hz}$, $1 \mathrm{H}), 2.00(\mathrm{~m}, 1 \mathrm{H}), 1.85(\mathrm{~m}, 2 \mathrm{H}), 1.13(\mathrm{~s}, 3 \mathrm{H}), 0.93(\mathrm{~s}, 6 \mathrm{H}), 0.91(\mathrm{~s}, 3 \mathrm{H}), 0.89(\mathrm{~s}, 3 \mathrm{H}), 0.74(\mathrm{~s}$, $3 \mathrm{H}), 0.72(\mathrm{~s}, 3 \mathrm{H}) .\left[{ }^{13} \mathrm{C}\right] \mathrm{NMR}(101 \mathrm{MHz}, \mathrm{DMSO}-\mathrm{d} 6) \delta 177.20,144.10,132.10,123.00,118.20$, $113.50,92.50,81.50,76.80,74.10,71.50,66.50,62.20,55.70,47.70,47.10,46.40,42.20$, $42.10,39.90,39.10,38.80,37.90,34.20,33.10,32.70,30.90,29.00,26.90,26.90,26.00$, $25.50,24.00,23.80,23.70,23.70,18.60,17.30,16.20$.

Oleanolic 3-O- $\beta$-D-mannopyranoside $(1 \mathrm{~m}): 16.2 \%$ yield, $\mathrm{R}_{\mathrm{f}}=0.22\left(10: 1 \mathrm{CH}_{2} \mathrm{Cl}_{2}-\right.$ $\mathrm{MeOH}) .\left[{ }^{1} \mathrm{H}\right] \mathrm{NMR}(400 \mathrm{MHz}, \mathrm{DMSO}-\mathrm{d} 6) \delta 12.00(\mathrm{~s}, 1 \mathrm{H}), 5.16(\mathrm{~s}, 1 \mathrm{H}), 4.77(\mathrm{~s}, 1 \mathrm{H}), 4.71^{2}(\mathrm{~d}$, $\mathrm{J}=4.5 \mathrm{~Hz}, 1 \mathrm{H}), 4.66(\mathrm{~d}, \mathrm{~J}=4.0 \mathrm{~Hz}, 1 \mathrm{H}), 4.53(\mathrm{~d}, \mathrm{~J}=5.6 \mathrm{~Hz}, 1 \mathrm{H}), 4.37(\mathrm{~s}, 1 \mathrm{H}), 3.61(\mathrm{~m}, 1 \mathrm{H})$, 3.51 (br s, 1H), 3.49-3.37 (m, 2H), 3.22-3.15 (m, 2H), $3.06(\mathrm{~m}, 1 \mathrm{H}), 2.74(\mathrm{~d}, \mathrm{~J}=12.1 \mathrm{~Hz}, 1 \mathrm{H})$, 1.96-1.86 (m, 1H), $1.81(\mathrm{~m}, 2 \mathrm{H}), 1.10(\mathrm{~s}, 3 \mathrm{H}), 0.95(\mathrm{~s}, 3 \mathrm{H}), 0.88(\mathrm{~s}, 9 \mathrm{H}), 0.73(\mathrm{~s}, 3 \mathrm{H}), 0.72(\mathrm{~s}$, $3 \mathrm{H}) .\left[{ }^{13} \mathrm{C}\right] \mathrm{NMR}(101 \mathrm{MHz}, \mathrm{DMSO}-\mathrm{d} 6) \delta 178.54,143.85,121.44,95.86,80.26,74.46,71.16$, $71.04,66.89,61.34,54.90,47.03,45.68,45.43,41.33,40.79,38.89,37.89,37.49,36.54$, $33.30,32.80,32.36,32.07,30.37,28.51,27.17,25.58,23.35,22.91,22.60,21.07,17.84$, $16.84,16.56,15.07$.

Oleanolic - 28 -allyl ester-3-O- $\alpha$-L-rhamnopyranoside (1f): $87.3 . \%$ yield, $\mathrm{R}_{\mathrm{f}}=0.44$ $\left(10: 1 \mathrm{CH}_{2} \mathrm{Cl}_{2}-\mathrm{MeOH}\right) .\left[{ }^{1} \mathrm{H}\right] \mathrm{NMR}\left(400 \mathrm{MHz}, \mathrm{CDCl}_{3}\right) \delta 5.90$ (ddd, J = 22.8, 10.8, 5.6 Hz, $\left.1 \mathrm{H}\right)$, 
$5.32(\mathrm{dd}, 1 \mathrm{H}), 5.29(\mathrm{~s}, 1 \mathrm{H}), 5.21(\mathrm{dd}, \mathrm{J}=10.5 \mathrm{~Hz}, 1 \mathrm{H}), 4.80(\mathrm{~s}, 1 \mathrm{H}), 4.52(\mathrm{~m}, 2 \mathrm{H}), 3.92(\mathrm{dd}$, 1H), $3.76(\mathrm{~m}, 2 \mathrm{H}), 3.41(\mathrm{~m}, 1 \mathrm{H}), 3.09$ (dd, J = 11.3, $4.8 \mathrm{~Hz}, 1 \mathrm{H}), 2.88(\mathrm{~d}, \mathrm{~J}=9.6 \mathrm{~Hz}, 1 \mathrm{H}), 1.96$ (m, 1H), $1.87(\mathrm{~m}, 2 \mathrm{H}), 1.26(\mathrm{~s}, 3 \mathrm{H}), 1.13(\mathrm{~s}, 3 \mathrm{H}), 0.93(\mathrm{~s}, 3 \mathrm{H}), 0.92(\mathrm{~s}, 3 \mathrm{H}), 0.90(\mathrm{~s}, 6 \mathrm{H}), 0.76$ (s, 3H), $0.72(\mathrm{~s}, 3 \mathrm{H}) \cdot\left[{ }^{13} \mathrm{C}\right]$ NMR (101 MHz, DMSO-d6) $\delta$ 177.20, 144.10, 132.10, 123.00, $118.20,113.70,92.50,74.20,74.10,73.70,72.40,66.50,55.40,47.70,47.10,46.40,42.20$, $42.10,39.90,39.10,38.80,37.90,34.20,33.10,32.70,30.90,29.00,26.90,26.90,26.00$, $25.50,24.00,23.80,23.70,23.70,18.60,17.30,17.00,16.20$.

Oleanolic -3-O- $\alpha$-L-rhamnopyranoside $(1 \mathrm{n}): 48.0 \%$ yield, $\mathrm{R}_{\mathrm{f}}=0.35\left(10: 1 \mathrm{CH}_{2} \mathrm{Cl}_{2}-\mathrm{MeOH}\right)$.

Oleanolic -28 -heptenyl ester-3-O- $\beta$-D-galactopyranoside $(1 \mathrm{~g}): 82.0 \%$ yield, $\mathrm{R}_{\mathrm{f}}=0.29$ (10:1 $\left.\mathrm{CH}_{2} \mathrm{Cl}_{2}-\mathrm{MeOH}\right) .\left[{ }^{1} \mathrm{H}\right] \mathrm{NMR}(400 \mathrm{MHz}, \mathrm{DMSO}-\mathrm{d} 6) \delta 5.77(\mathrm{~m}, 1 \mathrm{H}), 5.18(\mathrm{~s}, 1 \mathrm{H}), 4.96(\mathrm{~m}$, $2 \mathrm{H}), 4.70(\mathrm{~s}, 1 \mathrm{H}), 4.59(\mathrm{~s}, 1 \mathrm{H}), 4.48(\mathrm{~s}, 1 \mathrm{H}), 4.28(\mathrm{~s}, 1 \mathrm{H}), 4.10(\mathrm{~d}, \mathrm{~J}=6.6 \mathrm{~Hz}, 1 \mathrm{H}), 3.93(\mathrm{~m}, 2 \mathrm{H})$, $3.62(\mathrm{~s}, 1 \mathrm{H}), 3.52(\mathrm{~m}, 1 \mathrm{H}), 3.41(\mathrm{~m}, 1 \mathrm{H}), 3.27(\mathrm{~m}, 3 \mathrm{H}), 3.02(\mathrm{~d}, \mathrm{~J}=7.9 \mathrm{~Hz}, 1 \mathrm{H}), 2.79(\mathrm{~d}, \mathrm{~J}=12.5$ $\mathrm{Hz}, 1 \mathrm{H}), 1.98(\mathrm{~m}, 3 \mathrm{H}), 1.80(\mathrm{~m}, 3 \mathrm{H}), 1.10(\mathrm{~s}, 3 \mathrm{H}), 0.98(\mathrm{~s}, 3 \mathrm{H}), 0.88(\mathrm{~s}, 6 \mathrm{H}), 0.86(\mathrm{~s}, 3 \mathrm{H}), 0.75(\mathrm{~s}$, $3 \mathrm{H}), 0.66(\mathrm{~s}, 3 \mathrm{H}) . \quad\left[{ }^{13} \mathrm{C}\right] \mathrm{NMR}(101 \mathrm{MHz}$, DMSO-d6) $\delta 176.50,143.44,138.47,121.84,114.70$, $106.02,87.87,74.82$, 73.56, 71.10, 68.00, 63.56, 60.29, 55.01, 46.99, 46.00, 45.40, 41.20, 40.89, $38.89,38.68,38.10,36.24,33.18,33.11,32.70,32.32,32.09,30.33,27.90,27.78,27.62,27.05$, $25.63,25.53,25.05, \mathrm{~h} 23.29,22.90,22.53,17.75,16.67,16.43,15.03$.

Oleanolic -28 - heptenyl ester-3-O- $\alpha$-D-arabinopyranoside $(1 \mathrm{~h}): 85.0 \%$ yield, $\mathrm{R}_{\mathrm{f}}=$ $0.41\left(10: 1 \mathrm{CH}_{2} \mathrm{Cl}_{2}-\mathrm{MeOH}\right) .\left[{ }^{1} \mathrm{H}\right] \mathrm{NMR}(400 \mathrm{MHz}, \mathrm{DMSO}-\mathrm{d} 6) \delta 5.78(\mathrm{~m}, 1 \mathrm{H}), 5.18(\mathrm{~s}, 1 \mathrm{H})$, $4.96(\mathrm{~m}, 2 \mathrm{H}), 4.79(\mathrm{~d}, \mathrm{~J}=3.8 \mathrm{~Hz}, 1 \mathrm{H}), 4.49(\mathrm{~d}, \mathrm{~J}=4.9 \mathrm{~Hz}, 1 \mathrm{H}), 4.46(\mathrm{~d}, \mathrm{~J}=3.8 \mathrm{~Hz}, 1 \mathrm{H}), 4.11$ $(\mathrm{d}, \mathrm{J}=5.8 \mathrm{~Hz}, 1 \mathrm{H}), 3.93(\mathrm{~m}, 2 \mathrm{H}), 3.71-3.54(\mathrm{~m}, 2 \mathrm{H}), 3.38-3.24(\mathrm{~m}, 3 \mathrm{H}), 3.00(\mathrm{~d}, \mathrm{~J}=10.7 \mathrm{~Hz}$, $1 \mathrm{H}), 2.79(\mathrm{~d}, \mathrm{~J}=13.4 \mathrm{~Hz}, 1 \mathrm{H}), 1.99(\mathrm{~m}, 3 \mathrm{H}), 1.80(\mathrm{~m}, 2 \mathrm{H}), 1.72(\mathrm{~d}, \mathrm{~J}=12.0 \mathrm{~Hz}, 1 \mathrm{H}), 1.10(\mathrm{~s}$, $3 \mathrm{H}), 0.97(\mathrm{~s}, 3 \mathrm{H}), 0.88(\mathrm{~s}, 6 \mathrm{H}), 0.86(\mathrm{~s}, 3 \mathrm{H}), 0.76(\mathrm{~s}, 3 \mathrm{H}), 0.66(\mathrm{~s}, 3 \mathrm{H}) .\left[{ }^{13} \mathrm{C}\right] \mathrm{NMR}(101 \mathrm{MHz}$, DMSO-d6) $\delta 176.51,143.43,138.47,121.83,114.71,105.87,87.64,72.69,70.97,67.61$, $65.09,63.57,54.91,46.96,46.01,45.40,41.21,40.90,38.88,38.74,38.02,36.25,33.16$, $33.11,32.70,32.30,32.09,30.33,27.90,27.78,27.58,27.04,25.62,25.52,25.05,23.29$, $22.89,22.53,17.74,16.67,16.39,15.04$.

\section{Synthesis of oleanolic28-glycosides}

$\beta$-D-glucopyranosyl oleanolate (2a): OA (200 mg,0.44 mmol), benzoylbromoglucoside (Glc-6) $(320 \mathrm{mg}, 0.49 \mathrm{mmol}), 10 \mathrm{mg}$ tetrabutyl ammonium bromide, and $300 \mathrm{mg}$ potassium carbonate were dissolved in a mixture of $\mathrm{CH}_{2} \mathrm{Cl}_{2} / \mathrm{H}_{2} \mathrm{O}(10 / 0.5 \mathrm{~mL})$. The mixture was stirred vigorously for $6 \mathrm{~h}$ under reflux. The reaction mixture was diluted with $\mathrm{CHCl}_{3}$ and washed successively with water, diluted in $0.5 \mathrm{~mol} \mathrm{HCl}$, and saturated with $\mathrm{NaCl}$. The organic layer was separated and concentrated. The residue was subjected to silica gel column chromatography (4:1, petroleum ether-EtOAc) to afford benzoylglucosyl-28-oleanolate (OAglc-12) (360 mg, $0.35 \mathrm{mmol}, 79.5 \%$ yield) as a white solid. $\mathrm{R}_{\mathrm{f}}=0.24$ (3:1 petroleum etherEtOAc). OA-glc-12 (300 mg, $0.29 \mathrm{mmol})$ was dissolved in a solution of $\mathrm{CH}_{2} \mathrm{Cl}_{2}-\mathrm{MeOH}(1: 1)$ and $20 \mathrm{mg} \mathrm{NaOMe}$ was added. The mixture was stirred for $2 \mathrm{~h}$ at room temperature. The filtrate was concentrated and purified with a silica gel column chromatography (20:1 $\rightarrow 10: 1$, $\mathrm{CH}_{2} \mathrm{Cl}_{2}-\mathrm{MeOH}$ ) to give $\beta$-D-glucopyranosyl oleanolate(2a) (140 mg, $0.23 \mathrm{mmol}, 79.3 \%$ yield), $\mathrm{R}_{\mathrm{f}}=0.25\left(10: 1, \mathrm{CH}_{2} \mathrm{Cl}_{2}-\mathrm{MeOH}\right) .{ }^{1} \mathrm{H}$ NMR (400 MHz, DMSO) $\delta 5.24(\mathrm{~d}, J=8.0 \mathrm{~Hz}$, $1 \mathrm{H}, \mathrm{OH}-2^{\prime}$ ), 5.17 (s, 1H,H-12), 4.96 (brs, 2H,0H-3', OH-4') 4.41 (brs, 1H,OH-6'), 4.28 (d, $J=$ $\left.4.0 \mathrm{~Hz}, 1 \mathrm{H}, \mathrm{H}-1^{\prime}\right), 3.62$ (d, $\left.J=10.6 \mathrm{~Hz}, 1 \mathrm{H}, \mathrm{H}-6^{\prime}-1\right), 3.44$ (m,1H,H-6'-2), 3.20 (m,1H,H-3'), 3.12 (m, 3H,H-4',H-5',H-2'), 2.99 (m, 1H,H-3), 2.74 (d, $J=10.6 \mathrm{~Hz}, 1 \mathrm{H}, \mathrm{H}-18), 1.95$ (t, $J=11.9 \mathrm{~Hz}$,

Genetics and Molecular Research 15 (3): gmr.15038998 
1H,H-16-1), 1.78 (m,3H,H-2-1,H-11), 1.08 (s, 3H,H-27), 0.89 (s, 3H,H-23), 0.88 (s, 3H,H29), 0.87 (s, 3H,H-30), 0.85 (s, 3H,H-25), 0.69 (s, 3H,H-24), 0.68 (s, 3H,H-26).

Compounds $\mathbf{2 b - 2 f}$ were prepared according to the same procedure described for $\mathbf{2 a}$. $\left[{ }^{1} \mathrm{H}\right] \mathrm{NMR}$ and $\left[{ }^{13} \mathrm{C}\right]$ NMR data of $2 \mathrm{c}, 2 \mathrm{~b}, 2 \mathrm{f}$ are identical with literature reports (Li et al., 2009; Zhao et al., 2011).

$\beta$-D-galactopyranosyl oleanolate (2c): $68.0 \%$ yield, $\mathrm{R}_{\mathrm{f}}=0.25\left(10: 1, \mathrm{CH}_{2} \mathrm{Cl}_{2}-\mathrm{MeOH}\right)$. $\beta$-D-xylopyranosyl oleanolate (2b): $75.0 \%$ yield, $\mathrm{R}_{\mathrm{f}}=0.35\left(10: 1, \mathrm{CH}_{2} \mathrm{Cl}_{2}-\mathrm{MeOH}\right)$.

$\alpha$-L-arabinopyranosyl oleanolate (2d): $68.0 \%$ yield, $\mathrm{R}_{\mathrm{f}}=0.35\left(10: 1, \mathrm{CH}_{2} \mathrm{Cl}_{2}-\mathrm{MeOH}\right)$. $\left[{ }^{1} \mathrm{H}\right] \mathrm{NMR}(400 \mathrm{MHz}, \mathrm{DMSO}) \delta 5.36\left(\mathrm{~d}, \mathrm{~J}=4.5 \mathrm{~Hz}, 1 \mathrm{H}, \mathrm{OH}-2^{\prime}\right), 5.20$ (brs, $\left.1 \mathrm{H}, \mathrm{H}-12\right), 5.16(\mathrm{~d}$, $\left.\mathrm{J}=4.9 \mathrm{~Hz}, 1 \mathrm{H}, 0 \mathrm{H}-3^{\prime}\right), 4.66\left(\mathrm{~m}, 1 \mathrm{H}, \mathrm{OH}-4^{\prime}\right), 4.28\left(\mathrm{~d}, \mathrm{~J}=5.0 \mathrm{~Hz}, 1 \mathrm{H}, \mathrm{H}-1^{\prime}\right), 3.77$ - $3.66(\mathrm{~m}, 1 \mathrm{H}, \mathrm{H}-$ 5'-1), 3.55 (brs, 1H,H-4'), 3.49 (t, 1H,H-2'), 3.38 (m, 1H,H-3'), 3.17 (d, J = 5.0 Hz, 1H,H-5' -2), 3.04 - 2.95 (m, 1H,H-3), 2.82 (d, J = 10.4 Hz, 1H,H-18), 1.95 (m, 1H,H-16-1), 1.80 (d, $\mathrm{J}=5.7 \mathrm{~Hz}, 2 \mathrm{H}, \mathrm{H}-11), 1.09$ (s, 3H,H-27), 0.89 (s,3H,H-23), 0.88 (s, 6H,H-29,H-30), 0.85 (s, 3H,H-25), 0.68 (s, 6H,H-24,H-26). [ $\left.{ }^{13} \mathrm{C}\right]$ NMR (101 MHz, DMSO-d6) $\delta 175.29,143.47$, $121.78,93.76,76.81,71.22,69.59,65.52,63.50,54.81,47.08,46.10,45.47,41.23,40.64$, $38.66,38.36,38.11,36.55,33.22,32.77,32.34,31.74,30.36,28.21,27.15,26.94,25.57$, $23.36,22.93,22.26,17.96,16.67,16.01,15.11$.

$\beta$-D-mannopyranosyl oleanolate (2e): $22.0 \%$ yield, $\mathrm{R}_{\mathrm{f}}=0.24\left(10: 1, \mathrm{CH}_{2} \mathrm{Cl}_{2}-\mathrm{MeOH}\right)$. $\left[{ }^{1} \mathrm{H}\right] \mathrm{NMR}(400 \mathrm{MHz}, \mathrm{DMSO}) \delta 5.81(\mathrm{~s}, 1 \mathrm{H}), 5.19(\mathrm{~d}, \mathrm{~J}=17.1 \mathrm{~Hz}, 1 \mathrm{H}), 5.08(\mathrm{t}, \mathrm{J}=6.3 \mathrm{~Hz}$, $1 \mathrm{H}), 4.91(\mathrm{t}, \mathrm{J}=5.1 \mathrm{~Hz}, 1 \mathrm{H}), 4.73(\mathrm{~d}, \mathrm{~J}=4.5 \mathrm{~Hz}, 1 \mathrm{H}), 4.49(\mathrm{t}, \mathrm{J}=6.0 \mathrm{~Hz}, 1 \mathrm{H}), 4.28(\mathrm{~d}, \mathrm{~J}=5.1$ $\mathrm{Hz}, 1 \mathrm{H}), 4.02$ (dd, J = 6.8, 3.6 Hz, 1H), $3.55-3.47$ (m, 1H), $3.00(\mathrm{~d}, \mathrm{~J}=5.2 \mathrm{~Hz}, 1 \mathrm{H}), 2.91-$ $2.65(\mathrm{~m}, 1 \mathrm{H}), 1.98(\mathrm{dd}, \mathrm{J}=16.6,10.0 \mathrm{~Hz}, 1 \mathrm{H}), 1.82(\mathrm{~s}, 1 \mathrm{H}), 1.10(\mathrm{~s}, 1 \mathrm{H}), 0.89(\mathrm{~s}, 3 \mathrm{H}), 0.85(\mathrm{~s}$, $1 \mathrm{H}), 0.68$ (s, 2H). $\left[{ }^{13} \mathrm{C}\right] \mathrm{NMR}(101 \mathrm{MHz}$, DMSO-d6) $\delta$ 174.76, 143.22, 122.01, 93.32, 76.79, $76.68,70.83,69.30,66.10,60.66,54.71,47.04,46.38,45.28,41.25,40.89,38.73938 .89$, $38.34,38.10,36.51,33.05,32.69,32.25,31.82,30.36,28.20,26.93,26.93,25.52,23.28$, $22.98,22.54,17.99,16.75,15.97,15.06$.

$\alpha$-L-rhamnopyranosyl oleanolate (2f): $80.0 \%$ yield, $\mathrm{R}_{\mathrm{f}}=0.36\left(10: 1, \mathrm{CH}_{2} \mathrm{Cl}_{2}-\mathrm{MeOH}\right)$.

\section{Synthesis of oleanolic -3, 28-diglycosides}

3-O- $\beta$-D-glucopyranosyl-28-O- $\beta$-D-glucopyranosyloleanolate(3a):Trichloroacetimidate donor Glc-8 (0.5 g, $0.68 \mathrm{mmol})$ and intermediate OA-glc-12 ( $0.6 \mathrm{~g}, 0.58 \mathrm{mmol})$ were dissolved in dry $10 \mathrm{~mL} \mathrm{CH} \mathrm{Cl}_{2}$. The solution was added to a $1.0 \mathrm{~g} 4-\AA$ molecular sieve and stirred at room temperature for $20 \mathrm{~min}$. The reaction temperature was reduced to $0{ }^{\circ} \mathrm{C}$, TMSOTf $(10 \mu \mathrm{L}$, $0.055 \mathrm{mmol}$ ) was added dropwise and stirred for $2 \mathrm{~h}$ at room temperature. The reaction was stopped by dropwise adding triethylamine, according to the reaction process detected by TLC (4:1, petroleum ether-ethyl acetate). The reaction solution was concentrated and subjected to column chromatography to afford a white solid OA-3,28-glc-13 (0.75 g, $0.46 \mathrm{mmol})$ with a rate of $79.3 \%$. $\mathrm{R}_{\mathrm{f}}=0.22$ (3:1, petroleum ether-ethyl acetate).

OA-3,28-glc-13 was dissolved in $20 \mathrm{~mL} \mathrm{1:1} \mathrm{MeOH}-\mathrm{CH}_{2} \mathrm{Cl}_{2}$ solution. $\mathrm{NaOMe}(20$ $\mathrm{mg}$ ) was added and stirred for $4 \mathrm{~h}$ at room temperature. The mixture was neutralized with acidic cation exchange resin to $\mathrm{pH} 7$ and filtered according to the process detected by TLC (5:1, $\left.\mathrm{CH}_{2} \mathrm{Cl}_{2}-\mathrm{MeOH}\right)$. The filtrate was concentrated and purified with a silica gel column chromatography $\left(20: 1 \rightarrow 10: 1, \mathrm{CH}_{2} \mathrm{Cl}_{2}-\mathrm{MeOH}\right)$ to give a white solid 3a (Sha et al., 2008) $(0.25$ $\mathrm{g}, 0.32 \mathrm{mmol}$ ) with $69.6 \%$ yield (total $55.2 \%$ yield) and $\left.\mathrm{R}_{\mathrm{f}}=0.29\left(5: 1, \mathrm{CH}_{2} \mathrm{Cl}_{2}-\mathrm{MeOH}\right) .{ }^{1} \mathrm{H}\right]$ NMR (400 MHz, DMSO-d6) $\delta 5.23(\mathrm{~d}, \mathrm{~J}=8.0 \mathrm{~Hz}, 1 \mathrm{H}), 5.16(\mathrm{~s}, 1 \mathrm{H}), 5.13(\mathrm{~d}, \mathrm{~J}=5.7 \mathrm{~Hz}, 1 \mathrm{H})$,

Genetics and Molecular Research 15 (3): gmr.15038998 
$4.99(\mathrm{~d}, \mathrm{~J}=4.5 \mathrm{~Hz}, 1 \mathrm{H}), 4.93(\mathrm{~d}, \mathrm{~J}=4.5 \mathrm{~Hz}, 1 \mathrm{H}), 4.83(\mathrm{~m}, 3 \mathrm{H}), 4.40(\mathrm{t}, \mathrm{J}=5.3 \mathrm{~Hz}, 1 \mathrm{H}), 4.33$ (t, J $=5.3 \mathrm{~Hz}, 1 \mathrm{H}), 4.09(\mathrm{dd}, \mathrm{J}=10.4,5.1 \mathrm{~Hz}, 1 \mathrm{H}), 3.62(\mathrm{~m}, 2 \mathrm{H}), 3.43(\mathrm{~m}, 2 \mathrm{H}), 3.22-3.06(\mathrm{~m}, 5 \mathrm{H}), 3.04$ (m, 3H), 2.98-2.91 (m, 1H), $2.74(\mathrm{~d}, \mathrm{~J}=12.7 \mathrm{~Hz}, 1 \mathrm{H}), 1.93(\mathrm{~m}, 1 \mathrm{H}), 1.80(\mathrm{~m}, 2 \mathrm{H}), 1.08(\mathrm{~s}, 3 \mathrm{H})$, 0.97 (s, 3H), 0.87 (s, 9H), 0.75 (s, 3H), 0.68 (s, 3H). $\left[{ }^{13} \mathrm{C}\right] \mathrm{NMR}(100 \mathrm{MHz}$, DMSO-d6) $\delta$ 175.20, 143.47, 121.67, 105.37, 94.09, 87.93, 77.71, 76.91, 76.68, 76.57, 73.98, 72.38, 70.17, 69.58, 61.21, $60.71,55.08,47.13,45.93,45.56,41.27,40.76,38.99,38.71,38.24,36.29,33.25,32.75,32.29$, $31.61,30.31,27.66,27.19,25.52,25.52,23.36,22.96,22.52,17.76,16.68,16.49,15.20$.

Compounds $\mathbf{3 b} \mathbf{b} \mathbf{3}$ e were prepared according to the same procedure described for $\mathbf{3 a}$.

3-O- $\beta$-D-xylopyranosyl-28-O-a-L-arabinopyranosyl oleanolate (3b): $62.0 \%$ yield, $\mathrm{R}_{\mathrm{f}}$ $\left.=0.62\left(5: 1, \mathrm{CH}_{2} \mathrm{Cl}_{2}-\mathrm{MeOH}\right) .{ }^{1} \mathrm{H}\right] \mathrm{NMR}(400 \mathrm{MHz}, \mathrm{DMSO}-\mathrm{d} 6) \delta 5.36(\mathrm{~d}, \mathrm{~J}=4.5 \mathrm{~Hz}, 1 \mathrm{H})$, $5.19(\mathrm{~s}, 1 \mathrm{H}), 5.14(\mathrm{~d}, \mathrm{~J}=4.8 \mathrm{~Hz}, 1 \mathrm{H}), 4.87(\mathrm{br} \mathrm{s}, 3 \mathrm{H}), 4.65(\mathrm{~s}, 1 \mathrm{H}), 4.61(\mathrm{~s}, 1 \mathrm{H}), 4.11(\mathrm{~d}, \mathrm{~J}=7.5$ $\mathrm{Hz}, 1 \mathrm{H}), 3.71(\mathrm{~m}, 2 \mathrm{H}), 3.64(\mathrm{dd}, \mathrm{J}=11.3,5.3 \mathrm{~Hz}, 1 \mathrm{H}), 3.54(\mathrm{~m}, 1 \mathrm{H}), 3.51-3.46(\mathrm{~m}, 1 \mathrm{H}), 3.36$ $(\mathrm{d}, \mathrm{J}=8.5 \mathrm{~Hz}, 1 \mathrm{H}), 3.24(\mathrm{~m}, 1 \mathrm{H}), 3.01(\mathrm{~m}, 4 \mathrm{H}), 2.81(\mathrm{~d}, \mathrm{~J}=10.3 \mathrm{~Hz}, 1 \mathrm{H}), 1.92(\mathrm{~m}, 1 \mathrm{H}), 1.78$ (m, 2H), $1.09(\mathrm{~s}, 3 \mathrm{H}), 0.97(\mathrm{~s}, 3 \mathrm{H}), 0.88(\mathrm{~s}, 6 \mathrm{H}), 0.86(\mathrm{~s}, 3 \mathrm{H}), 0.75(\mathrm{~s}, 3 \mathrm{H}), 0.68(\mathrm{~s}, 3 \mathrm{H}) \cdot\left[{ }^{13} \mathrm{C}\right]$ NMR (101 MHz, DMSO-d6) $\delta$ 175.24, 143.42, 121.72, 106.11, 93.74, 87.60, 76.73, 73.73, $71.19,69.57,69.57,65.53,65.48,63.44,54.86,46.98,46.07,45.44,41.21,40.61,38.90$, $38.72,38.05,36.23,33.20,32.71,32.27,31.70,30.31,27.49,27.12,25.69,25.48,23.33$, $22.89,22.24,17.69,16.64,16.36,15.08$.

3-O- $\beta$-D-galactopyranosyl-28-O-a-L-arabinopyranosyl oleanolate (3c): $60.0 \%$ yield, $\left.\mathrm{R}_{\mathrm{f}}=0.41\left(5: 1, \mathrm{CH}_{2} \mathrm{Cl}_{2}-\mathrm{MeOH}\right) .{ }^{1} \mathrm{H}\right] \mathrm{NMR}(400 \mathrm{MHz}, \mathrm{DMSO}-\mathrm{d} 6) \delta 5.37(\mathrm{~s}, 1 \mathrm{H}), 5.20(\mathrm{~s}, 1 \mathrm{H})$, $5.12(\mathrm{~s}, 1 \mathrm{H}), 4.68-4.56(\mathrm{~m}, 3 \mathrm{H}), 4.54(\mathrm{~s}, 1 \mathrm{H}), 4.45(\mathrm{~s}, 1 \mathrm{H}), 4.24(\mathrm{~s}, 1 \mathrm{H}), 4.11(\mathrm{~d}, 1 \mathrm{H}), 3.70$ (m, 2H), $3.63(\mathrm{~s}, 1 \mathrm{H}), 3.59-3.46(\mathrm{~m}, 3 \mathrm{H}), 3.45-3.34(\mathrm{~m}, 2 \mathrm{H}), 3.29(\mathrm{~m}, 3 \mathrm{H}), 3.04(\mathrm{~d}, 1 \mathrm{H}), 2.82$ $(\mathrm{d}, \mathrm{J}=11.5 \mathrm{~Hz}, 1 \mathrm{H}), 1.93(\mathrm{~m}, 1 \mathrm{H}), 1.80(\mathrm{~s}, 2 \mathrm{H}), 1.09$ (s, 3H), 0.98 (s, 3H), $0.88(\mathrm{~s}, 9 \mathrm{H}), 0.76$ (s, 3H), $0.68(\mathrm{~s}, 3 \mathrm{H}) . \quad\left[{ }^{13} \mathrm{C}\right] \mathrm{NMR}(101 \mathrm{MHz}, \mathrm{DMSO}-\mathrm{d} 6) \delta$ 175.30, 143.48, 121.78, 106.00, 93.77, 87.89, 74.85, 73.56, 71.24, 71.12, 69.59, 68.06, 65.55, 63.54, 60.36, 55.04, 47.04, $46.10,45.47,41.21,40.64,38.74,38.70,38.16,36.25,33.22,32.77,32.30,31.75,30.37$, 27.66, 27.16, 25.65, 25.54, 23.37, 22.93, 22.26, 17.75, 16.68, 16.48, 15.13 .

3-O-a-L-arabinopyranosyl-28-O- $\beta$-D-galactopyranosyl oleanolate $(3 \mathrm{~d}): 70.0 \%$ yield, $\left.\mathrm{R}_{\mathrm{f}}=0.50\left(5: 1, \mathrm{CH}_{2} \mathrm{Cl}_{2}-\mathrm{MeOH}\right) .{ }^{1} \mathrm{H}\right] \mathrm{NMR}(400 \mathrm{MHz}, \mathrm{DMSO}-\mathrm{d} 6) \delta 5.21(\mathrm{~d}, \mathrm{~J}=7.8 \mathrm{~Hz}, 1 \mathrm{H})$, $5.16(\mathrm{~s}, 1 \mathrm{H}), 4.94(\mathrm{~d}, 1 \mathrm{H}), 4.76(\mathrm{~m}, 2 \mathrm{H}), 4.54(\mathrm{~s}, 1 \mathrm{H}), 4.50-4.42(\mathrm{~m}, 3 \mathrm{H}), 4.11(\mathrm{~m}, 1 \mathrm{H}), 3.67(\mathrm{br}$ s, $1 \mathrm{H}), 3.63$ (br s, 1H), 3.60 (br s, 1H), $3.50(\mathrm{~m}, 1 \mathrm{H}), 3.45-3.30(\mathrm{~m}, 7 \mathrm{H}), 3.00(\mathrm{~d}, \mathrm{~J}=10.5 \mathrm{~Hz}$, $1 \mathrm{H}), 2.75(\mathrm{~d}, \mathrm{~J}=12.8 \mathrm{~Hz}, 1 \mathrm{H}), 1.93(\mathrm{~m}, 1 \mathrm{H}), 1.78(\mathrm{~s}, 2 \mathrm{H}), 1.08(\mathrm{~s}, 3 \mathrm{H}), 0.96(\mathrm{~s}, 3 \mathrm{H}), 0.87(\mathrm{~s}$, 9H), 0.76 (s, 3H), 0.68 (s, 3H). $\left[{ }^{13} \mathrm{C}\right] \mathrm{NMR}(101 \mathrm{MHz}, \mathrm{DMSO}-\mathrm{d} 6) \delta$ 175.26, 143.42, 121.63, $105.77,94.59,87.68,75.88,73.38,72.68,70.99,69.45,67.72,67.56,65.00,59.90,54.98$, $47.06,45.90,45.55,41.25,40.72,38.96,38.74,38.10,36.27,33.22,32.72,32.25,31.60$, $30.27,27.62,27.15,25.63,25.51,23.35,22.92,22.49,17.75,16.66,16.41,15.15$.

3-O- $\beta$-D-galactopyranosyl-28-O- $\beta$-D-galactopyranosyl oleanolate $(3 \mathrm{e}): 72.0 \%$ yield, $\mathrm{R}_{\mathrm{f}}=0.29\left(5: 1, \mathrm{CH}_{2} \mathrm{Cl}_{2}-\mathrm{MeOH}\right) .\left[{ }^{1} \mathrm{H}\right] \mathrm{NMR}(400 \mathrm{MHz}, \mathrm{DMSO}-\mathrm{d} 6) \delta 5.21(\mathrm{~d}, \mathrm{~J}=7.8 \mathrm{~Hz}, 1 \mathrm{H})$, $5.16(\mathrm{~s}, 1 \mathrm{H}), 4.97(\mathrm{~d}, \mathrm{~J}=5.6 \mathrm{~Hz}, 1 \mathrm{H}), 4.78(\mathrm{~d}, \mathrm{~J}=4.9 \mathrm{~Hz}, 1 \mathrm{H}), 4.70(\mathrm{~d}, 1 \mathrm{H}), 4.60(\mathrm{~d}, 1 \mathrm{H}), 4.56$ (t, 1H), $4.50(\mathrm{~m}, 2 \mathrm{H}), 4.29(\mathrm{~d}, \mathrm{~J}=3.8 \mathrm{~Hz}, 1 \mathrm{H}), 4.10(\mathrm{~d}, \mathrm{~J}=5.6 \mathrm{~Hz}, 1 \mathrm{H}), 3.68$ (br s, 1H), 3.61 (br s, 1H), $3.51(\mathrm{~m}, 2 \mathrm{H}), 3.45-3.20(\mathrm{~m}, 8 \mathrm{H}), 3.03(\mathrm{~d}, \mathrm{~J}=11.0 \mathrm{~Hz}, 1 \mathrm{H}), 2.75(\mathrm{~d}, \mathrm{~J}=12.4 \mathrm{~Hz}$, 1H), $1.93(\mathrm{~m}, 1 \mathrm{H}), 1.78(\mathrm{~m}, 3 \mathrm{H}), 1.08(\mathrm{~s}, 3 \mathrm{H}), 0.98(\mathrm{~s}, 3 \mathrm{H}), 0.87(\mathrm{~s}, 6 \mathrm{H}), 0.86(\mathrm{~s}, 3 \mathrm{H}), 0.75$ (s, 3H), $0.68(\mathrm{~s}, 3 \mathrm{H}) . \quad\left[{ }^{13} \mathrm{C}\right] \mathrm{NMR}(101 \mathrm{MHz}, \mathrm{DMSO}-\mathrm{d} 6) \delta$ 175.27, 143.44, 121.62, 105.94, $94.58,87.87,75.88,74.84,73.55,73.41,71.13,69.44,68.06,67.70,60.36,59.87,55.04$, $47.06,45.89,45.52,41.22,40.70,38.96,38.68,38.16,36.24,33.19,32.70,32.21,31.60$, $30.27,27.63,27.13,25.60,25.49,23.33,22.89,22.46,17.71,16.65,16.45,15.13$.

Genetics and Molecular Research 15 (3): gmr.15038998 


\section{Antifungal activity bioassay}

Antifungal activities of the target compounds against $M$. oryzae were assessed by the mycelium growth rate method (Agricultural Industry Standard of the People's Republic of China, 2006). Each synthetic saponin $(100 \mathrm{mg} / \mathrm{mL})$, isoprothiolane $(0.11,0.78,1.56,3.13$, $6.25,12.50,25.00 \mathrm{mg} / \mathrm{mL})$ as a positive control, and a negative control $(0 \mathrm{mg} / \mathrm{mL})$ in PDA were mixed thoroughly and sterilized $\left(121^{\circ} \mathrm{C}, 1.2 \mathrm{~atm}, 30 \mathrm{~min}\right)$, then poured separately into sterilized Petri dishes and allowed to solidify. Three 8-mm-diameter plugs of actively growing M. oryzae mycelium colonies were placed in a triangular shape onto each plate (replicated, two plates, six plugs per treatment). The plates were incubated at $27^{\circ} \mathrm{C}$. The colony diameter and the radial mycelium growth were measured on the fourth day.

Diameter of mycelia and inhibition rate were calculated using the following formula:

$$
I=\frac{\overline{D_{0}}-\overline{D_{1}}}{\overline{D_{0}}} \times 100 \%
$$

where I is the inhibition rate, $\overline{D_{0}}$ is the average diameter of mycelia in the blank test, and $\overline{D_{1}}$ is the average diameter of mycelia in the presence of synthetic saponins or isoprothiolane. The inhibition rates of these compounds at $100 \mu \mathrm{g} / \mathrm{mL}$ are shown in Table 1.

Table 1. The structure, inhibitory rate, $\mathrm{R}_{\mathrm{f}}$ and yield of synthetic saponins $(100 \mu \mathrm{g} / \mathrm{mL})$ on Magnaporthe oryzae.

\begin{tabular}{|c|c|c|c|c|c|c|}
\hline No. & Compound & Yield (\%) & $\mathrm{R}_{\mathrm{f}}$ & $\mathrm{C}-3(\mathrm{OH})$ & $\mathrm{C}-28(\mathrm{COOH})$ & $I(\%)$ \\
\hline 1 & 1a(Glc) & 83.8 & 0.26 & $-\beta$-D-Glc & $-\mathrm{CH}_{2} \mathrm{CH}=\mathrm{CH}_{2}$ & $60.52 \pm 1.52$ \\
\hline 2 & $1 \mathrm{c}(\mathrm{Gal})$ & 58.4 & 0.26 & - $\beta$-D-Gal & $-\mathrm{CH}_{2} \mathrm{CH}=\mathrm{CH}_{2}$ & $63.44 \pm 1.51$ \\
\hline 3 & $1 \mathrm{~b}(\mathrm{Xyl})$ & 76.4 & 0.36 & $-\beta-\mathrm{D}-\mathrm{Xyl}$ & $-\mathrm{CH}_{2} \mathrm{CH}=\mathrm{CH}_{2}$ & $6.24 \pm 0.92$ \\
\hline 4 & $1 \mathrm{~d}($ Ara $)$ & 83.5 & 0.38 & $-\alpha$-L-Ara & $-\mathrm{CH}_{2} \mathrm{CH}=\mathrm{CH}_{2}$ & $8.56 \pm 1.14$ \\
\hline 5 & 1e(Man) & 33.8 & 0.25 & - $\alpha$-D-Man & $-\mathrm{CH} 2 \mathrm{CH}=\mathrm{CH} 2$ & $75.46 \pm 0.61$ \\
\hline 6 & 1f(Rha) & 87.3 & 0.44 & $-\alpha$-L-Rha & $-\mathrm{CH}_{2} \mathrm{CH}=\mathrm{CH}_{2}$ & $27.29 \pm 3.64$ \\
\hline 7 & $1 \mathrm{i}(\mathrm{Glc})$ & 46.7 & 0.21 & $-\beta$-D-Glc & $\mathrm{H}$ & $41.29 \pm 1.14$ \\
\hline 8 & $1 \mathrm{k}(\mathrm{Gal})$ & 32.2 & 0.22 & $-\beta$-D-Gal & $\mathrm{H}$ & $32.43 \pm 4.12$ \\
\hline 9 & $1 \mathrm{j}(\mathrm{Xyl})$ & 45.0 & 0.33 & $-\beta$-D-Xyl & $\mathrm{H}$ & $13.29 \pm 2.69$ \\
\hline 10 & 11(Ara) & 48.0 & 0.34 & $-\alpha$-L-Ara & $\mathrm{H}$ & $16.31 \pm 1.32$ \\
\hline 11 & $1 \mathrm{~m}$ (Man) & 16.2 & 0.22 & $-\alpha-D-M a n$ & $\mathrm{H}$ & $48.24 \pm 4.85$ \\
\hline 12 & $\ln$ (Rha) & 48.0 & 0.35 & $-\alpha$-L-Rha & $\mathrm{H}$ & $15.21 \pm 1.82$ \\
\hline 13 & $1 \mathrm{~g}(\mathrm{Gal})$ & 82.0 & 0.29 & $-\beta$-D-Gal & $-\left(\mathrm{CH}_{2}\right)_{5} \mathrm{CH}=\mathrm{CH}_{2}$ & $40.14 \pm 6.31$ \\
\hline 14 & $1 \mathrm{~h}$ (Ara) & 85.0 & 0.41 & $-\alpha-L-A r a$ & $-\left(\mathrm{CH}_{2}\right)_{5} \mathrm{CH}=\mathrm{CH}_{2}$ & $35.17 \pm 0.40$ \\
\hline 15 & $2 \mathrm{a}(\mathrm{Glc})$ & 79.3 & 0.25 & $\mathrm{H}$ & $-\beta$-D-Glc & $14.40 \pm 5.30$ \\
\hline 16 & $2 \mathrm{c}(\mathrm{Gal})$ & 68.0 & 0.25 & $\mathrm{H}$ & $-\beta$-D-Gal & $23.77 \pm 7.00$ \\
\hline 17 & $2 \mathrm{~b}(\mathrm{Xyl})$ & 75.0 & 0.35 & $\mathrm{H}$ & $-\beta$-D-Xyl & $13.60 \pm 1.98$ \\
\hline 18 & $2 \mathrm{~d}(\mathrm{Ara})$ & 68.0 & 0.35 & $\mathrm{H}$ & $-\alpha$-L-Ara & $16.41 \pm 2.87$ \\
\hline 19 & 2e(Man) & 22.0 & 0.24 & $\mathrm{H}$ & $-\beta$-D-Man & $39.48 \pm 5.34$ \\
\hline 20 & $2 \mathrm{f}(\mathrm{Rha})$ & 80.0 & 0.36 & $\mathrm{H}$ & $-\alpha-\mathrm{L}-\mathrm{Rha}$ & $25.08 \pm 3.15$ \\
\hline 21 & $3 \mathrm{a}(\mathrm{Glc}, \mathrm{Glc})$ & 55.2 & 0.29 & $-\beta$-D-Glc & $-\beta$-D-Glc & $16.97 \pm 3.60$ \\
\hline 22 & 3b(Xyl,Ara) & 62.0 & 0.62 & $-\beta-\mathrm{D}-\mathrm{Xyl}$ & $-\alpha$-L-Ara & $34.21 \pm 0.84$ \\
\hline 23 & $3 \mathrm{c}(\mathrm{Gal}, \mathrm{Ara})$ & 60.0 & 0.41 & $-\beta$-D-Gal & $-\alpha-\mathrm{L}-\mathrm{Ara}$ & $31.88 \pm 4.28$ \\
\hline 24 & $3 \mathrm{~d}(\mathrm{Ara}, \mathrm{Gal})$ & 70.0 & 0.50 & $-\alpha-L-A r a$ & $-\beta-\mathrm{D}-\mathrm{Gal}$ & $9.79 \pm 1.49$ \\
\hline 25 & $3 \mathrm{e}(\mathrm{Gal}, \mathrm{Gal})$ & 72.0 & 0.29 & $-\beta$-D-Gal & $-\beta$-D-Gal & $38.76 \pm 2.65$ \\
\hline Ck1 & $\mathrm{OA}$ & & & $\mathrm{H}$ & $\mathrm{H}$ & $14.00 \pm 1.49$ \\
\hline Ck2 & OA-10a & 82.6 & & $\mathrm{H}$ & $-\mathrm{CH} 2 \mathrm{CH}=\mathrm{CH} 2$ & $12.99 \pm 1.98$ \\
\hline Ck3 & $\mathrm{OA}-10 \mathrm{~b}$ & & & $\mathrm{H}$ & $-(\mathrm{CH} 2) 5 \mathrm{CH}=\mathrm{CH} 2$ & $20.68 \pm 2.52$ \\
\hline Ck4 & \multicolumn{3}{|c|}{ Isoprothiolane $(\mu \mathrm{g} / \mathrm{mL})$} & \multicolumn{3}{|c|}{$1.30 \pm 1.32(0.11)$ to $84.82 \pm 2.64(12.50)$} \\
\hline
\end{tabular}

Genetics and Molecular Research 15 (3): gmr.15038998 


\section{RESULTS AND DISCUSSION}

\section{Synthesis of oleanolic mono- and di-glycosides}

\section{Synthesis of oleanolic $C_{3}$ monoglycosides}

The two-methyl groups at the C-4 position in OA make the hydroxy C-3 glycosylation reaction difficult; a glycosyl donor with high activity is therefore required. Trichloroacetimidate glycosyl as a donor has the advantages of high activity, high stability, and a single spatial structure that can efficiently catalyze glycosylation reactions promoted by trimethylsilyl trifluoromethanesulfinate (TMSOTf). During the glycosylation reaction, other hydroxyl groups in the saccharide-ring must to be operated through protection. The common protection group contains benzyl, alkyl, acetyl, and benzoyl. We chose benzoyl-protected trichloroacetimidate as a glycosyl donor for glycosylation because of its high stability and neighbor-participating effect (Deng et al., 1999), and a glycosyl product with a single beta space configuration (Figure 1).

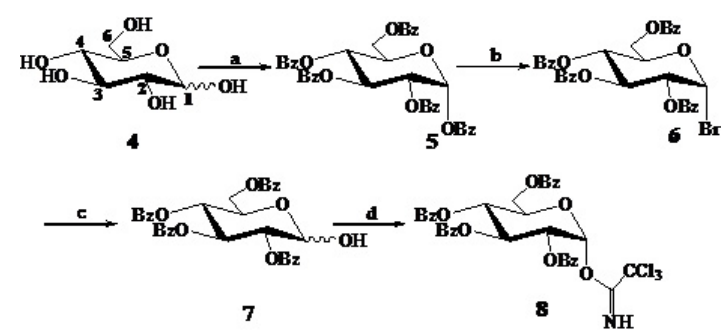

Figure 1. The synthetic routes of glycosyl bromide and trichloroacetimidates donors. Reagen s and conditions: (a) $\mathrm{BzCl}$, pyridine, $\mathrm{rt}, 24 \mathrm{~h}, 95 \%$ yield; (b) $\mathrm{HBr}-\mathrm{HOAc}, \mathrm{CH}_{2} \mathrm{Cl}_{2}$, rt, $2 \mathrm{~h}$; (c) $\mathrm{Ag}_{2} \mathrm{CO}_{3}$, acetone- $\mathrm{H}_{2} \mathrm{O}, \mathrm{rt}, 2 \mathrm{~h}, 92.6 \%$ yield; (d) $\mathrm{CCl}_{3} \mathrm{CN}, \mathrm{DBU}, \mathrm{CH}_{2} \mathrm{Cl}_{2}, \mathrm{rt}, 4 \mathrm{~h}, 84.4 \%$ yield.

Activity against $M$. oryzae was higher with oleanolic 3-monoglycosides containing allyl at the $\mathrm{C}_{28}$-carboxyl position than without the protection group (Table 1). A similar report showed that the activity of an inhibiting HIV proteolytic enzyme was enhanced after oleanolic $\mathrm{C}_{28}$-carboxyl was introduced, and an optimal chain length of 6-8 carbon atoms was used (Ma et al., 2000). Therefore, a heptenyl with seven carbon atoms was available to link oleanolic $\mathrm{C}_{28}{ }^{-}$ carboxyl before oleanolic $\mathrm{C}_{3}$-hydroxy glycosylation with galactose and arabinose, consequently affording saponins with an oleanolic 3-monosaccharide 28-heptenyl ester (Figures 2 and 3).

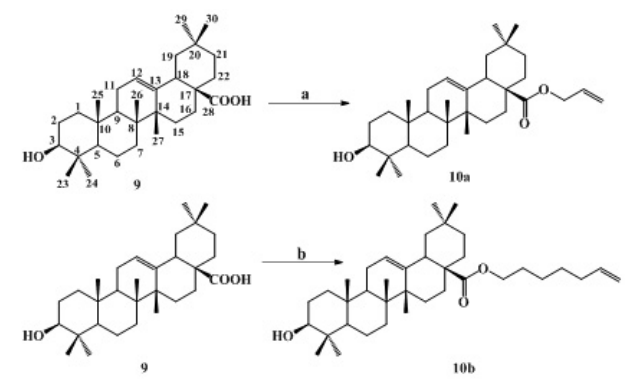

Figure 2. Synthesis of glycosylacceptor. Reagents and conditions: a. Allyl Br, DBU, DMF, rt, 24h; b. 7-bromo-1heptene, DBU, DMF, rt, 24h.

Genetics and Molecular Research 15 (3): gmr.15038998 

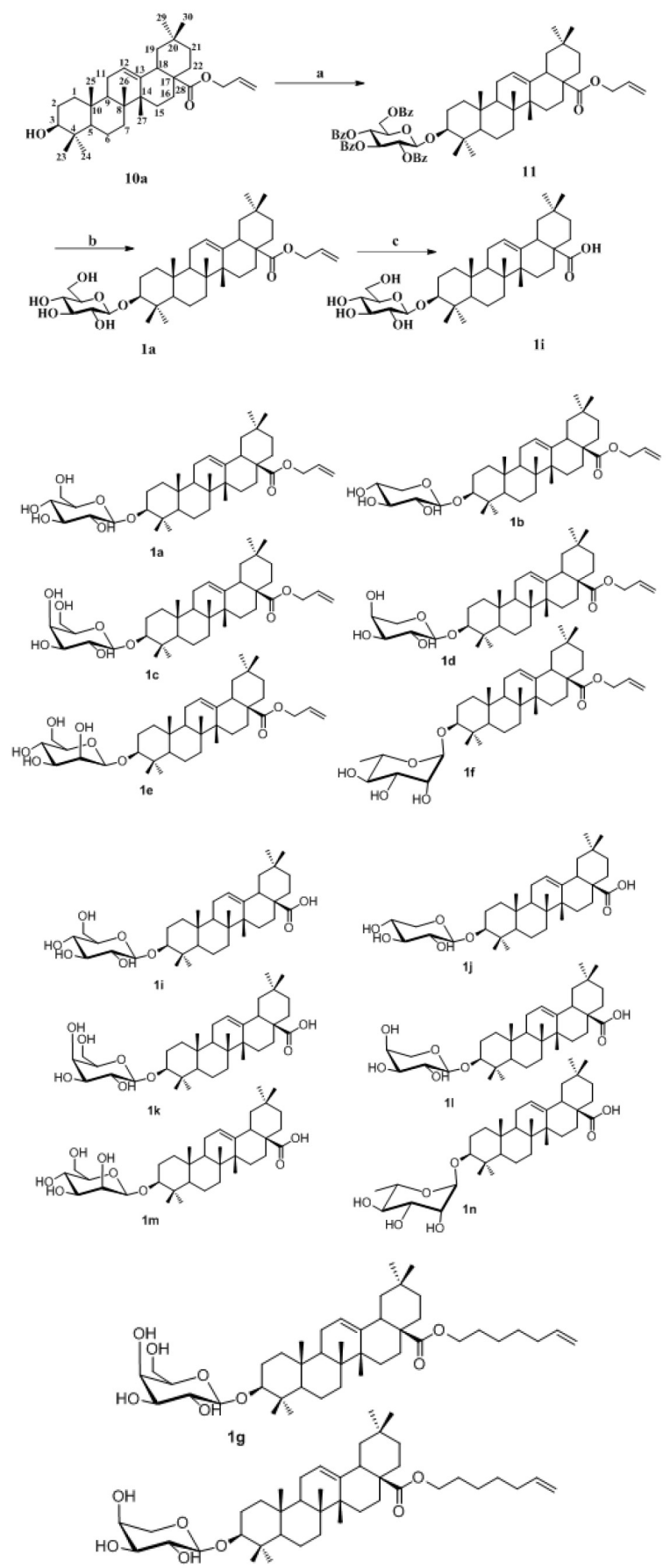

$1 \mathrm{~h}$

Figure 3. The synthetic routes and their products of oleanolic3-glycosides. Reagents and conditions: a. TMSOTf, $0^{\circ} \mathrm{C}, 4 \AA \mathrm{MS}, \mathrm{CH}_{2} \mathrm{Cl}_{2}, 4$ h; b. $\mathrm{MeONa}, \mathrm{CH}_{2} \mathrm{Cl}_{2}-\mathrm{MeOH}$, rt, 2 h; c. $\mathrm{PdCl}_{2}, \mathrm{CH}_{3} \mathrm{OH}$, rt, 24 h. 
Oleanolic 3-monoglycosides and 3-monoglycosides 28-allyl or heptenyl ester were verified by $\left[{ }^{1} \mathrm{H}\right],\left[{ }^{13} \mathrm{C}\right]$ NMR data analysis. The $\left[{ }^{1} \mathrm{H}\right]$ NMR data displayed olefinic $\mathrm{C}_{12}-\mathrm{H}$ at $\delta 5.29 \mathrm{br} \mathrm{s}$ in $\mathrm{CDCl}_{3}$ or $\delta 5.16 \mathrm{~s}$ in DMSO-d $\mathrm{d}_{6}, \mathrm{C}_{3}-\mathrm{H}$ at $\delta 3.14 \mathrm{~m}$ in $\mathrm{CDCl}_{3}$ or $\delta 3.02 \mathrm{~m}$ in DMSO-d6, $\mathrm{C}_{18}-\mathrm{H}$ at $\delta 2.88 \mathrm{~d}$ in $\mathrm{CDCl}_{3}$ or $\delta 2.74 \mathrm{~d}$ in DMSO-d6; seven $\mathrm{H}$ from $-\mathrm{CH}_{3}$ at $\delta 0.72 \mathrm{~s}-1.16 \mathrm{~s}$; $\delta 5.32 \mathrm{dd}, \delta 4.90 \mathrm{ddd}, \delta 4.53 \mathrm{~m} ; \mathrm{C}_{28}$-allyl at $\delta 5.32 \mathrm{dd}, \delta 4.90 \mathrm{ddd}, \delta 4.53 \mathrm{~m}$ while $\mathrm{C}_{28}$-heptenyl at $\delta 5.78 \mathrm{~m}, \delta 4.96 \mathrm{~m}, \delta 3.93 \mathrm{~m}$ or $\mathrm{C}_{28}-\mathrm{OOH}$ at $\delta 12.00 \mathrm{~s}$; anomeric proton at $\delta 4.32 \mathrm{~d}(\beta-\mathrm{Glc}), \delta 4.11 \mathrm{~d}$ ( $\beta$-Gal), $\delta 4.11 \mathrm{~d}(\beta-\mathrm{Xyl}), \delta 4.12 \mathrm{~d}(\alpha-\mathrm{Ara}), \delta 4.37 \mathrm{~s}(\alpha-\mathrm{Man}), \delta 4.66 \mathrm{~s}(\alpha-\mathrm{Rha})$. The $\left[{ }^{13} \mathrm{C}\right] \mathrm{NMR}$ data showed 36 carbons at saponin Glc-9, Gal-9, Man-9, Rha-9 and 35 carbons at saponin Xyl9, Ara-9; carboxyl $\mathrm{C}_{28}$ at $\delta 178.5$ while the carbon signal of $\mathrm{C}_{28}$-heptenyl at $\delta 138.47, \delta 114.70$, 863.56, d33.11, $827.90, \delta 27.78, \delta 25.05 ; \mathrm{C}_{13}=\mathrm{C}_{12}$ at $\delta 143.8, \delta 121.5 ; \mathrm{C}_{3}$ at $880.26-87.95$; anomeric carbon at $\delta 105.44$ ( $\beta$-Glc), $\delta 106.12$ ( $\beta$-Gal), $\delta 106.18$ ( $\beta$-Xyl), $\delta 105.85$ ( $\alpha$-Ara), $\delta 95.86$ ( $\alpha$-Man), $\delta 102.85$ ( $\alpha$-Rha). These NMR data are consistent with those in previous reports (Zang, 2004; Sha et al., 2008; Zhao et al., 2011).

\section{Synthesis of oleanolic 28 monoglycosides}

The usual strategy for preparing oleanolic $\mathrm{C}_{28}$ glycosyl ester involves two methods. In the first method, trichloroacetimidate glycoside as glycosyl donor is coupled with oleanolic $\mathrm{C}_{28}$-carboxyl after $\mathrm{C}_{3}$-hydroxyl is protected by acetylation. Then an additional deprotection step is performed to provide oleanolic $\mathrm{C}_{28}$ monoglycoside. The second method adopts the "phasetransfer-catalyzed method" (Bliard et al., 1994) where bromoglycoside as a glycosyl donor is coupled with $\mathrm{OA} \mathrm{C}_{28}$-carboxyl promoted by potassium carbonate-tetrabutylammonium bromide in a two-phase water-chloroform solution. Oleanolic $\mathrm{C}_{3}$ hydroxyl in such conditions does not participate in the reaction and therefore, there is no need to protect in advance. It has been reported that the second method is simpler, higher-yielding, and better than the first method (Yan, 2008), therefore the second method was adopted in this study (Figure 4).

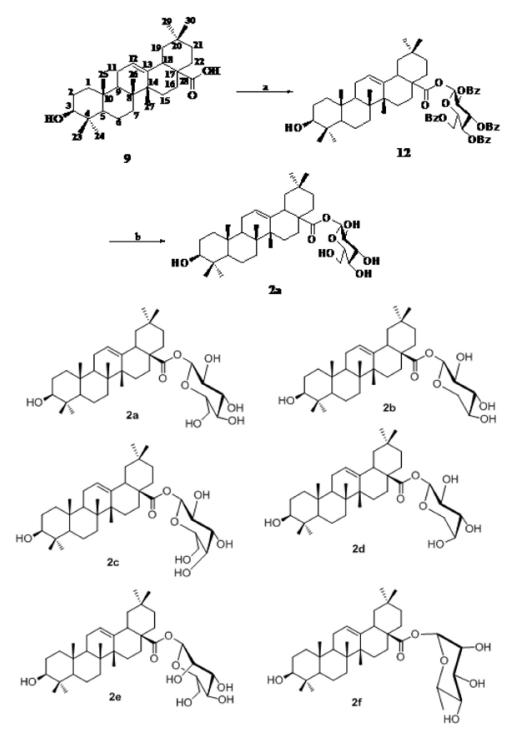

Figure 4. The synthetic routes and their products of oleanolic 28-glycosides. Reagents and conditions: a. $\mathrm{K}_{2} \mathrm{CO}_{3}$, $\mathrm{Bu}_{4} \mathrm{NBr}, \mathrm{CH}_{2} \mathrm{Cl}_{2}-\mathrm{H}_{2} \mathrm{O}$, reflux, 6 h. b. $\mathrm{CH}_{3} \mathrm{ONa}, \mathrm{CH}_{2} \mathrm{Cl}_{2}-\mathrm{CH}_{3} \mathrm{OH}, \mathrm{rt}, 2 \mathrm{~h}$.

Genetics and Molecular Research 15 (3): gmr.15038998 
Oleanolic 28 monoglycosides were confirmed by $\left[{ }^{1} \mathrm{H}\right]-,\left[{ }^{13} \mathrm{C}\right]-\mathrm{NMR}$ data analysis. The $\left[{ }^{1} \mathrm{H}\right]$ NMR data demonstrated $\mathrm{C}_{12}-\mathrm{H}$ at $\delta 5.16 \mathrm{~s} ; \mathrm{C}_{3}-\mathrm{H}$ at $\delta 2.99 \mathrm{~m} ; \mathrm{C}_{3}-\mathrm{OH}$ at $\delta 4.27 \mathrm{~s} ; \mathrm{C}_{18}-\mathrm{H}$ at $\delta 2.74-2.82 \mathrm{~d} ; \mathrm{H}$ from seven $-\mathrm{CH}_{3}$ at $\delta 0.67 \mathrm{~s}-1.11 \mathrm{~s}$; anomeric proton at $\delta 5.24 \mathrm{~d}(\beta-\mathrm{Glc}), \delta 5.21 \mathrm{~d}$ ( $\beta$-Gal), $\delta 5.21 \mathrm{~d}(\beta-\mathrm{Xyl}), \delta 5.36 \mathrm{~d}(\alpha-A r a), \delta 5.81 \mathrm{~s}(\alpha-\mathrm{Man}), \delta 5.76 \mathrm{~s}(\alpha-\mathrm{Rha}), \delta 6.19 \mathrm{~d}$ and 5.95s $\left(\beta-\right.$ Mal in Pyr-d5). The $\left[{ }^{13} \mathrm{C}\right]$ NMR indicated 42 carbons in Mal-11, 36 carbons in Glc-11, Gal-11, Man-11, Rha-11, and 35 carbons in Xyl-11, Ara-11; carboxyl $\mathrm{C}_{28}$ at $8175.2 ; \mathrm{C}_{13}=\mathrm{C}_{12}$ at $\delta 143.4, \delta 121.6 ; \mathrm{C}_{3}$ linked hydroxyl at $\delta 76.8$ while anomeric carbon at $\delta 94.05(\beta-\mathrm{Glc}), \delta 94.58$ ( $\beta$-Gal), $\delta 94.68$ ( $\beta$-Xyl), $\delta 93.76$ ( $\alpha$-Ara), $\delta 93.32$ ( $\alpha$-Man), $\delta 93.37$ ( $\alpha$-Rha), $\delta 102.97$ and 95.48 $(\beta-M a l$ in Pyr-d5). These NMR data are in accordance with previous reports (Li et al., 2009; Zhao et al., 2011).

\section{Synthesis of oleanolic 3, 28 diglycosides}

The synthesis of oleanolic 3,28 diglycosides must take into consideration which site is glycosylated first. There are more complex routes in the first glycosylation of oleanolic $\mathrm{C}_{3}$ hydroxyl than $\mathrm{C}_{28}$-carboxyl because the former bears the operation of protection and deprotection. In some situations, the former has obvious advantages. For example, in preparing a series of saponins with the same $\mathrm{C}_{3}$ glycosyl and different $\mathrm{C}_{28}$ glycosyl chains, this method can reduce workload and improve efficiency. Moreover, it can effectively avoid too much reaction damage to $\mathrm{C}_{28}$ containing sensitive groups that are coupled to the $\mathrm{C}_{28}$ glycosyl chain in later reactions. In short, the method should be selected according to the needs of the target compounds. The latter method was adopted for preparing five oleanolic diglycosides, i.e., glycosylation modification of the carboxyl $\mathrm{C}_{28}$ first, then of the hydroxyl $\mathrm{C}_{3}$, and finally the deprotecting group in the hydroxyl of the sugar ring giving oleanolic 3,28 diglycosides (Figure 5).

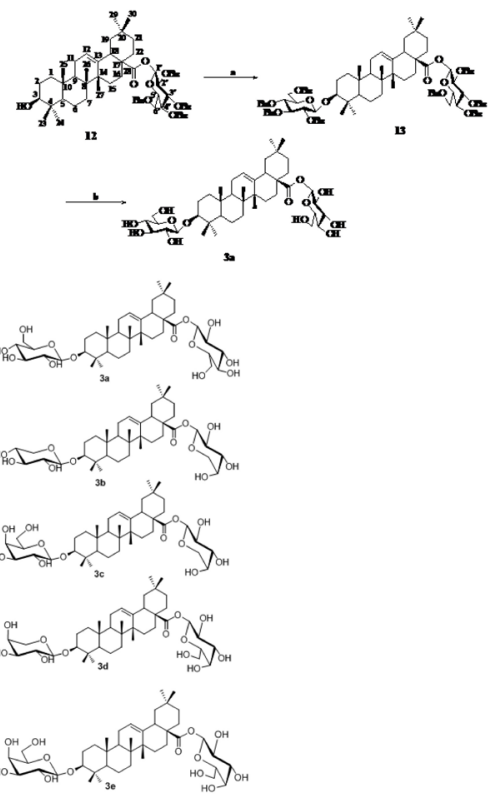

Figure 5. Synthetic routes and their products of oleanolic 3,28-disglycosides. Reagents and conditions: a. TMSOTf, $0^{\circ} \mathrm{C}, 4 \AA \mathrm{MS}, \mathrm{CH}_{2} \mathrm{Cl}_{2}, 4$ h; b. $\mathrm{CH}_{3} \mathrm{ONa}, \mathrm{CH}_{2} \mathrm{Cl}_{2}-\mathrm{CH}_{3} \mathrm{OH}, \mathrm{rt}, 2 \mathrm{~h}$.

Genetics and Molecular Research 15 (3): gmr.15038998 
Oleanolic 3, 28 diglycosides were demonstrated by $\left[{ }^{1} \mathrm{H}\right]-,\left[{ }^{13} \mathrm{C}\right]-\mathrm{NMR}$ data analysis. The $\left[{ }^{1} \mathrm{H}\right]$ NMR revealed $\mathrm{C}_{12}-\mathrm{H}$ at $\delta 5.16 \mathrm{~s} ; \mathrm{C}_{3}-\mathrm{H}$ at $\delta 3.00 \mathrm{~m} ; \mathrm{C}_{18}-\mathrm{H}$ at $\delta 2.74-2.82 \mathrm{~d} ; \mathrm{H}$ of seven $-\mathrm{CH}_{3}$ at $\delta 0.68 \mathrm{~s}-1.09 \mathrm{~s}$; proton signal linked anomeric carbon $\mathrm{C}_{3}$ and $\mathrm{C}_{28}$ at $\delta 4.09 \mathrm{~d}(\beta-\mathrm{Glc})$ and $\delta 5.23 \mathrm{~d}(\beta-\mathrm{Glc}), \delta 4.11 \mathrm{~d}(\beta-\mathrm{Xyl})$ and $\delta 5.36 \mathrm{~d}(\alpha-\mathrm{Ara}), \delta 4.11 \mathrm{~d}(\beta-\mathrm{Gal})$ and $\delta 5.37 \mathrm{~s}(\alpha-\mathrm{Ara}), \delta 4.11 \mathrm{~m}$ $(\alpha-\mathrm{Ara})$ and $\delta 5.21 \mathrm{~d}(\beta-\mathrm{Gal}), \delta 4.10 \mathrm{~d}(\beta-\mathrm{Gal})$ and $\delta 5.21 \mathrm{~d}(\beta-\mathrm{Gal})$ respectively. The $\left[{ }^{13} \mathrm{C}\right] \mathrm{NMR}$ brought out 42 carbons in saponin $3 \mathrm{a}$ and $3 \mathrm{e}, 41$ carbons in $3 \mathrm{c}$ and $3 \mathrm{~d}, 40$ carbon in $3 \mathrm{~b} ; \mathrm{C}_{28}$ at $\delta 175.20 ; \mathrm{C}_{13}=\mathrm{C}_{12}$ at $\delta 143.47$ and $\delta 121.67 ; \mathrm{C}_{3}$ at $\delta 87.60-87.93$ while anomeric carbon $\mathrm{C}_{3}$ and $\mathrm{C}_{28}$ at $\delta 105.37(\beta$-Glc) and $\delta 94.09$ ( $\beta$-Glc), $\delta 106.11$ ( $\beta$-Xyl) and $\delta 93.74(\alpha-A r a), \delta 106.00$ ( $\beta$-Gal) and $\delta 93.77(\alpha$-Ara), $\delta 105.77$ ( $\alpha$-Ara) and $\delta 94.59$ ( $\beta$-Gal), $\delta 105.94$ ( $\beta$-Gal) and $\delta 94.58$ $(\beta-\mathrm{Gal})$. These NMR data are consistent with those in previous reports (Zhao et al., 2011).

\section{Activity of oleanolic mono- and di-glycosides against M. oryzae and their SAR}

Twenty five oleanolic mono- and di-glycosides were synthesized, containing different structural characteristics: seven saccharide residues (pentose 2, hexose 4 and disaccharide $1)$, two linkage sites $\left(\mathrm{C}_{3}, \mathrm{C}_{28}\right)$, and aglycone $\mathrm{C}_{28}$ with or without a group such as allyl or heptenyl. These 25 synthesized oleanolic mono- or di-glycosides included fourteen oleanolic 3-monoglycosides, six oleanolic 28-monoglycosides, and five oleanolic 3-,28-diglycosides. Their inhibiting rates against $M$. oryzae at a concentrations of $100 \mu \mathrm{g} / \mathrm{mL}$ corresponded to $0.11-12.5 \mu \mathrm{g} / \mathrm{mL}$ for isoprothiolane (Table 1).

The SAR of the synthesized oleanolic monoglycosides showed significant differences in activity between different monosaccharide residues linked to $\mathrm{C}_{3}$ or $\mathrm{C}_{28}$ of $\mathrm{OA}$. In general, activity against $M$. oryzae of oleanolic hexose glycosides was stronger than one of the oleanolic pentose glycosides. The saccharide residue order of enhancing activity in oleanolic $\mathrm{C}_{3}$ monoglycosides (1i-1n) from strong to weak was mannose, glucose, and galactose, while rhamnose, arabinose, and xylose were ineffective. The saccharide residue order of enhancing activity in oleanolic $\mathrm{C}_{28}$ monoglycosides (2a-2f) from strong to weak was mannose, rhamnose, and galactose, while glucose, arabinose, and xylose were ineffective.

The glycosylation site in OA was another important factor affecting the activity of oleanolic monoglycosides. There was stronger activity in oleanolic $\mathrm{C}_{3}$ mannose, glucose, or galactose glycoside than in their $\mathrm{C}_{28}$ ester glycoside, but there was stronger activity in oleanolic $\mathrm{C}_{28}$ rhamnose ester glycoside than in its $\mathrm{C}_{3}$ glycoside. For oleanolic $\mathrm{C}_{3}$ or $\mathrm{C}_{28}$ pentose glycoside, arabinose, and xylose, they showed very little activity difference between the $\mathrm{C}_{3}$ and $\mathrm{C}_{28}$ sites.

Allyl and its length in $\mathrm{C}_{28}$ with a symmetrical $\mathrm{C}_{3}$ monosaccharide residue in OA obviously influenced their activity against $M$. oryzae. When $\mathrm{OA} \mathrm{C}_{28}$ was linked by allyl, the activity of oleanolic $\mathrm{C}_{3}$ hexose glycoside (1a,1c,1e,1f) was significantly enhanced. The hexose residue order of enhancing activity from strong to weak was galactose, mannose, glucose, and rhamnose. However, the activity of oleanolic $\mathrm{C}_{3}$ pentose glycosides with $\mathrm{C}_{28}$ allyl $(1 \mathrm{~b}, 1 \mathrm{~d})$ decreased to half that of aglycone $\mathrm{OA}$. When $\mathrm{OAC}_{28}$ was linked by heptenyl, the activity of both hexose and pentose glycosides in its $\mathrm{C}_{3}$ was increased (1g and $1 \mathrm{k}, 1 \mathrm{~h}$ and 11$)$. The activity enhanced in oleanolic hexose glycoside was less in $\mathrm{C}_{28}$ heptenyl than in $\mathrm{C}_{28}$ allyl (1 $\mathrm{g}$ and $1 \mathrm{c}$ ), while the activity enhanced in its pentose glycoside was more in $\mathrm{C}_{28}$ heptenyl than in $\mathrm{C}_{28}$ allyl (1h and 1d).

Activity of oleanolic diglycosides against $M$. oryzae was influenced by the saccharide residue type, linkage site, and a combination of both. When $\mathrm{C}_{3}$ and $\mathrm{C}_{28}$ of OA were linked to the same saccharide such as galactose or glucose, the activity linked to galactose (3e) was stronger than that linked to glucose (3a). When oleanolic $\mathrm{C}_{3}$ and $\mathrm{C}_{28}$ both were linked to a

Genetics and Molecular Research 15 (3): gmr.15038998 
pentose such as arabinose or xylose (3b), activity was stronger than when either $\mathrm{C}_{3}$ or $\mathrm{C}_{28}$ alone was linked to a pentose $(1 \mathrm{j}, 1 \mathrm{l} ; 2 \mathrm{~b}, 2 \mathrm{~d})$. When oleanolic $\mathrm{C}_{3}$ and $\mathrm{C}_{28}$ were linked to a different saccharide such as hexose galactose or pentose arabinose, the glycoside $\mathrm{C}_{3}$ linked a hexose than a pentose or the glycoside $\mathrm{C}_{28}$ linked a pentose than a hexose played more important role in activity $(3 \mathrm{c}, 3 \mathrm{~d})$.

In summary, the optimal oleanolic mono- and di-glycoside structure for activity against $M$. oryzae is a $\mathrm{C}_{3}$ connection of a hexose such as mannose, galactose, or glucose in combination with $\mathrm{a}_{28}$ connection to a small group such as allyl, or a $\mathrm{C}_{3}$ connection to a pentose accompanied by a larger group such as another pentose or heptenyl in $\mathrm{C}_{28}$.

\section{ACKNOWLEDGMENTS}

The authors would like to thank Professor Harald W. Scherm from the Department of Plant Pathology at the University of Georgia, USA for his helpful advice and proofreading the English style of our manuscript, and Dr. Satish Sreedharamurthy from the Department of Studies in Microbiology, University of Mysore, India for his literal advice. Research supported by the National Natural Sciences Foundation of China (grant \#21266010 to G. Huo), the Natural Sciences Fund of Jiangxi Province (grant \#20132BAB204028 to G. Huo), and China Scholarship Council Program (grant \#201308360006 to G. Huo).

\section{REFERENCES}

Agricultural Industry Standard of the People's Republic of China (2006). Indoor bioassay test criterion fungicides part 2: inhibiting pathogenic fungal hyphae growth test agar method. NY/T 1156.62006.

Bliard C, Massiot G and Nazabadioko S (1994). Glycosylation of acids under phase transfer conditions. Partial synthesis of saponins. Tetrahedron Lett. 35: 6107-6108. http://dx.doi.org/10.1016/0040-4039(94)88088-3.

Deng S, Yu B, Xie J and Hui Y (1999). Highly efficient glycosylation of sapogenins. J. Org. Chem. 64: 7265-7266. http:// dx.doi.org/10.1021/jo990603x

Fukuoka S, Saka N, Koga H, Ono K, et al. (2009). Loss of function of a proline-containing protein confers durable disease resistance in rice. Science 325: 998-1001. http://dx.doi.org/10.1126/science.1175550

Ghanbari R, Anwar F, Alkharfy KM, Gilani AH, et al. (2012). Valuable nutrients and functional bioactives in different parts of olive (Olea europaea L.)-a review. Int. J. Mol. Sci. 13: 3291-3340. http://dx.doi.org/10.3390/ijms13033291

Han YJ, Lin CZ, Wang QQ, Lu GD, et al. (2013). Expression investigation.of the putative chitinase family genes in $M$. oryzae. Redai Zuowu Xuebao 34: 1544-1551.

Huo GH, Zhang CL and Zhang YJ (2014). Structure elucidation of two triterpenoid saponins from leaves of Schima superba Gardn. et Champ. Lecture Notes Electrical Engineering, Springer 250: 915-922. http://dx.doi.org/10.1007/978-3642-37922-2_98

Li JF, Chen SJ, Zhao Y and Li JX (2009). Glycoside modification of oleanolic acid derivatives as a novel class of antiosteoclast formation agents. Carbohydr. Res. 344: 599-605. http://dx.doi.org/10.1016/j.carres.2009.01.019

Li XD, Kang ST, Xu HJ, Li GY, et al. (2011). Highly effective preparative method of glycosylation reagents-glycosyl trichloroacetimidates. J. Shenyang Pharm. Univ. 28: 707-711.

$\mathrm{Ma}$ CM, Nakamura N and Hattori M (2000). Chemical modification of oleanene type triterpenes and their inhibitory activity against HIV-1 protease dimerization. Chem. Pharm. Bull. 48: 1681-1688. http://dx.doi.org/10.1248/cpb.48.1681

Normile D (2009). Plant genetics. New strategy promises lasting resistance to a rice plague. Science 325: 925 . http:// dx.doi.org/10.1126/science.325 925

Sha Y, Yan MC, Liu J, Liu Y, et al. (2008). Facile synthesis of oleanolic acid monoglycosides and diglycosides. Molecules 13: 1472-1486. http://dx.doi.org/10.3390/molecules 13071472

Shi BJ and Wang GL (2008). Comparative study of genes expressed from rice fungus-resistant and susceptible lines during interactions with Magnaporthe oryzae. Gene 427: 80-85. http://dx.doi.org/10.1016/j.gene.2008.09.015

Sun PY, Liu JL, Wang Y, Jiang N, et al. (2013). Molecular mapping of the blast resistance gene Pi49 in the durably resistant rice cultivar Mowanggu. Euphytica 192: 45-54. http://dx.doi.org/10.1007/s10681-012-0829-3

Genetics and Molecular Research 15 (3): gmr.15038998 
Tan MH, Huo GH, Peng YM and Li F (2014). Efficacy and safety of blasticidin agent prepared by Schima superba, Sapindus mukorossi and other plants. Guangdong Agric. Sci. 16: 85-89.

Yan MC (2008). Design, synthesis and bioactivity investigation of oleanolic acid derivatives. Shenyang Pharmaceutical University.

Zang J (2004). Synthesis of oleanolic acid saponins. Master's thesis, Ocean University of China.

Zhang J, Huo GH, Li XM, Lan B, et al. (2013). The resistance of different strains of rice blast (M. oryzae) in Jiangxi Province to kasugamycin and isoprothiolane. Acta Agric. Univ. Jiangxiensis 35: 722-726.

Zhao H, Zong G, Zhang J, Wang D, et al. (2011). Synthesis and anti-fungal activity of seven oleanolic acid glycosides. Molecules 16: 1113-1128. http://dx.doi.org/10.3390/molecules16021113

Zheng LG, Liu Y, Liu YX, Wang GN, et al. (2005). Improvement of synthetic method of monosaccharide donor. $J$. Shenyang Pharm. Univ. 22: 352-354.

Zhou Y, Sheng YG, Zhao SZ, Qu L, et al. (2013). Determination of tricyclazole residues in cereals by LC-MS/MS method. J. Chin. Cereals Oils Assoc. 28: 102-105.

Zhu Y, Chen H, Fan J, Wang Y, et al. (2000). Genetic diversity and disease control in rice. Nature 406: 718-722. http:// dx.doi.org/10.1038/35021046

Genetics and Molecular Research 15 (3): gmr.15038998 